Recepción: 13 / 04 / 2018

Aceptación: 07 / 06 / 2018

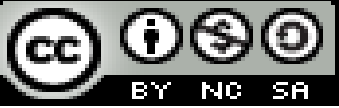

Ciencias de la Educación

Publicación: 02 / 07 / 2018

Artículo de investigación

\title{
La intervención comunitaria como parte del proceso de formación de los estudiantes de Trabajo Social
}

Community intervention as part of the process of formation of the students of Social Work

Intervenção da comunidade como parte do processo de formação de estudantes do Serviço Social

\author{
Marjorie M. Gómez-Zambrano ${ }^{\mathrm{I}}$ \\ marjorie.gomez@hotmail.com \\ Holger H. Góngora-Briones II \\ holger_07@hotmail.com
}

Correspondencia: marjorie.gomez@hotmail.com

\footnotetext{
I Magister en Trabajo Social, Licenciada en Trabajo Social, Trabajadora Social, Docente de la Universidad Laica "Eloy Alfaro" de Manabí, Manta, Ecuador.

II Licenciada en Trabajo Social, Universidad Laica "Eloy Alfaro” de Manabí, Manta, Ecuador.
} 


\section{Resumen}

En la carrera de Trabajo Social, la intervención social es una práctica que le posibilita al estudiante ubicar claramente su tendencia profesional acorde con su formación, desarrollar actitudes, habilidades y destrezas en los contextos donde le corresponde actuar. En este contexto, se desarrolló un trabajo cuyo objetivo fue establecer la importancia de la intervención comunitaria como parte del proceso de formación de los estudiantes de Trabajo Social. El mismo siguió como metodología de tipo cualitativa, a partir del método de sistematización. Como técnica para la recolección de la información, se utilizó la observación, las entrevistas grupales y la revisión documental, y como instrumentos el registro de experiencias y el diario de campo. La población la constituyeron los habitantes de la comuna "Ligüiqui". Entre sus resultados se pudo constatar que la comunidad de Ligüiqui tiene una organización que responde al modelo tradicional de conformación de los gobiernos locales y una población de aproximadamente 240 habitantes con el padecimiento de la diabetes. Como conclusión, se lograron actividades prácticas como producto de la intervención con las comunidades, generando nuevos conocimientos y competencias que desarrollaron los estudiantes de Trabajo Social.

Palabras clave: intervención comunitaria; formación de los estudiantes; trabajo social; comunidad.

\section{Abstract}

In Social Work career social intervention is a practice that allows the student clearly locate their professional trend consistent with their training, develop attitudes, skills, and abilities in the contexts where it belongs Act. In this context, developed a work whose objective was to establish the importance of the community as part of the process of formation of the students of Social work. The same followed as the methodology of qualitative type, from the method of systematization. As a technique for the collection of the information were observation, group interviews and documentary and as instruments review experiences registration and field journal. The inhabitants of the commune "Ligüiqui" constituted the population. Among its results it was found that the Liguiqui community has an organization that responds to the traditional model of formation of local governments and a population of approximately 240 people with the condition of diabetes. 
Conclusion achieved practical activities as a result of the intervention with communities, generating new knowledge and skills that students of social work developed.

Keywords: community-based intervention; training of students; social work; community.

\section{Resumo}

Na carreira do Serviço Social, a intervenção social é uma prática que possibilita ao aluno localizar claramente sua tendência profissional de acordo com sua formação, desenvolver atitudes, habilidades e habilidades nos contextos em que deve atuar. Nesse contexto, foi desenvolvido um trabalho cujo objetivo foi estabelecer a importância da intervenção comunitária como parte do processo de formação de alunos do Serviço Social. Seguiu-se como uma metodologia qualitativa, baseada no método de sistematização. Como técnica para coleta de informações, utilizou-se observação, entrevista em grupo e revisão documental, e como instrumentos o registro de experiências e o diário de campo. A população foi constituída pelos habitantes da comuna "Ligüiqui". Entre seus resultados constatou-se que a comunidade de Ligüiqui possui uma organização que responde ao modelo tradicional de conformação dos governos locais e uma população de aproximadamente 240 habitantes com a doença do diabetes. Como conclusão, atividades práticas foram alcançadas como resultado da intervenção com as comunidades, gerando novos conhecimentos e habilidades desenvolvidas pelos alunos do Serviço Social.

Palavras chave: intervenção comunitária; treinamento de estudantes; trabalho social; comunidade.

\section{Introducción}

Uno de los requisitos para poder titularse como Licenciado en Trabajo Social, es aprobar las 1200 horas de prácticas preprofesionales que se van distribuyendo en los 9 niveles de la carrera junto con la supervisión del tutor asignado; en cada nivel, los estudiantes realizan actividades diferentes y van complementándose de acuerdo a las materias que se estén cursando en el periodo académico actual. En los dos primeros semestres los talleres de prácticas son solo de observación, para que el estudiando vaya desarrollando una idea de lo que más adelante tendrá que trabajar, al respecto Costello (2011), expresa:

Como requisito previo a la obtención del título, los estudiantes deberán acreditar servicios a la comunidad, mediante Prácticas o Pasantías Pre-Profesionales, debidamente monitoreadas, en los 
campos de su especialidad de conformidad con los lineamientos generales definidos por el Consejo de Educación Superior (p.35).

Los estudiantes tienen un gran campo para realizar sus prácticas, en la carrera de Trabajo Social cuando se cursa en cuarto nivel se debe realizar un taller comunitario tal como dice la materia que está plasmada en la malla curricular vigente, por lo cual las prácticas se las debe realizar en cualquiera de las comunidades donde haya una facilidad para la intervención y un convenio con dicha comunidad, en caso de no haberlo se lo creará para que otros alumnos o los mismos regresen a continuar los proyectos ya establecidos, que en opinión de Costello (2011); "Dichas actividades se realizarán en Coordinación con el Vicerrectorado Académico, organizaciones comunitarias, empresas e instituciones públicas o privadas, relacionadas con la respectiva especialidad”. (p.67)

La Facultad tiene la obligación de designar un tutor para guiar, orientar y supervisar las actividades y proyectos que los estudiantes estén planificando o ejecutando en los campos de trabajo, junto con los respectivos tutores empresariales o representantes de las comunidades para la correcta aplicación de la práctica, y ese mismo docente deberá trabajar también con ellos en la materia de talleres para el correcto aprendizaje, partiendo desde lo teórico para luego ir al campo a aplicar lo que aprendió en el salón de clases, que en palabras de Costello (2011) se debe dar:

“Orientando, promoviendo y supervisando el vínculo entre la Universidad y las empresas e instituciones de la cadena productiva y de servicios, para reforzar mediante las Prácticas PreProfesionales, la formación de los estudiantes y su desempeño en el futuro”. (p.89)

En la carrera de Trabajo Social, las prácticas son un proceso que le posibilita al estudiante ubicar claramente su tendencia profesional acorde con su formación, desarrollar actitudes, habilidades y destrezas en los contextos donde le corresponde actuar. Además, le debe permitir al estudiante poner a prueba la solidez, coherencia y pertinencia de los enfoques teóricos, metodológicos e investigativos aplicables al objeto de conocimiento específico de estudio, y al énfasis del Trabajo Social, que es el espacio por excelencia en el cual el estudiante desarrolla competencias de trabajo con un equipo interdisciplinario necesarias para satisfacer las demandas del entorno.

Durante la ejecución de cualquier proyecto se generan toda una serie de aprendizajes y conocimientos, basados en las experiencias de participación de cada una de las personas y organizaciones que están involucradas (desde sus diferentes roles) en la gestión del proyecto. Tanto 
los estudiantes aprenden de la práctica comunitaria reforzando lo que se aprendió en la clase de forma teórica, mientras que el docente acompaña y supervisa la aplicación del conocimiento de alumno en el campo y en el salón de clases. "Ésta, es una metodología innovadora que parte de los aprendizajes de los participantes en un proceso para mejorar las prácticas del propio proyecto, así como extraer y aplicar lecciones aprendidas desde el punto de vista estratégico e institucional". (Economistas sin fronteras ATTAC, 2015, pp.45-78)

El hecho de sistematizar dichas experiencias y extraer de una forma participativa todos los aprendizajes, es fundamental para poder volcarlo en el propio proyecto y capitalizarlo en aprendizajes organizativos y participativos mismo que involucran a los actores sociales que participaron en la ejecución de tal proyecto, tanto docentes, alumnos y habitantes de la comunidad. "Cuando este conocimiento sistematizado, es adecuadamente gestionado, es decir, se encuentra con un contexto en el cual puede ser transmitido y aplicado, ocurre un proceso de socialización del conocimiento que daría paso a los aprendizajes organizativos". (Economistas sin fronteras ATTAC, 2015, pp.89-91)

En la realización del proyecto "Formación de un club de diabéticos en la comunidad de Ligüiqui", estuvo dirigido a las personas que padecen diagnóstico médico de esta enfermedad, con el fin de favorecer su conocimiento y compresión sobre los temas relacionados a la enfermedad de la diabetes y otras relacionadas a ellas, fortaleciendo el trabajo coordinado y en red de diferentes actores comunitarios (presidenta, doctores, pobladores) que trabajan en el desarrollo y mejoramiento de la comunidad.

Para la ejecución y gestión del proyecto, se estableció un convenio entre la Facultad de Trabajo Social y la directiva de la comuna de Ligüiqui, que permitió abarcar no solo la dimensión territorial y dar cobertura a las de 280 personas que están distribuidas por toda la comuna, sino fortalecer los conocimientos que le confiere al proyecto una mayor eficacia e impacto, para llevarlo a cabo se propuso como objetivo establecer la importancia de la intervención comunitaria como parte del proceso de formación de los estudiantes de Trabajo Social.

\section{Desarrollo}

La intervención comunitaria. El Trabajo Social es una disciplina que tiene muchas ramas de intervención, podemos decir que "La intervención comunitaria supone necesariamente trabajar 
desde los tres niveles de acción: la comunidad engloba a los distintos grupos que, a su vez, son configurados por distintas personas, sujetos de la intervención en sí”. (Rosello, 2010, p.47), que en base a la investigación que se realiza antes de intervenir, se detecta cuál de los subgrupos presenta la problemática que es el objeto de intervención con el cual el Trabajador Social desarrollará su trabajo.

Para trabajar en la problemática detectada, el Trabajador Social se sustenta en paradigmas, entre ellos el paradigma humanista ante esto Rosselló (2010), expresa que "Desde el paradigma humanista propuesto, les pueden resultar útiles estas aportaciones que deberán ser enriquecidas desde la práctica, a partir de la sistematización de un trabajo serio y riguroso" (p.56), debido a que se trabaja con los actores sociales (la comunidad) para con ellos mismos buscar la detección de las enigmas que les afecten y que ellos los actores que se encarguen de la resolución de las problemáticas, pero con la guía del Trabajador Social.

Desarrollo local. Dentro de los derechos que tienen los seres humanos como son el derecho a una vida digna, al trabajo, a la educación, etc., las personas también cuentan con derechos a la salud, a ser atendidos de una forma justa y digna en lugares donde deben recibir las medicinas y los cuidados para tener y garantizar una vida digna. "La Constitución del Ecuador del año 2008 define al Estado Ecuatoriano como uno de derechos y justicia, establece como primer deber del Estado el de respetar y garantizar la vigencia y tutela de los derechos humanos”. (Intriago, 2015, p.102)

En las comunidades donde la población es de bajos recursos económicos el Trabajador Social debe gestionar los medios y recursos necesarios junto con los habitantes, intentado hacerlo con un enfoque participativo, en donde los miembros de la comunidad se sientan actores de sus procesos de cambios. "El Trabajo Social, con comunidades es una línea de acción orientada hacia la consecución de determinados objetivos y supone la existencia de un centro de decisión dotado de los medios de intervención necesarios”. (Gómez, 2016, p.29)

Es de señalar, que la mayoría de los proyectos que se ejecutan en las comunidades rurales, son realizados por estudiantes universitarios, los cuales no van con un enfoque de tratar de cambiar la realidad social de esas poblaciones, más bien acuden con la obligación de aprobar la materia y dejar implementado un trabajo a medio hacer, con la finalidad de ganarse una nota y pasar el semestre. "El reconocimiento de la acción necesaria para intervenir en el terreno social y la consiguiente 
intervención concreta, del deseo de resolver una dificultad determinada, resulta de móviles muy variados y no siempre desinteresados”. (Gómez, 2016, p.44)

Comunidad. Cuando se interviene en comunidades pequeñas el profesional debe conocer todo el territorio y los patrones culturales de sus habitantes es importante que pueda adaptarse a ellos y ganar su confianza, para que al momento de recolectar información las personas le comenten con la mayor veracidad, aprendiendo desde lo práctico, pero ya con conocimiento teórico. "Desarrollo local es también una práctica y un saber de fronteras para poner en valor los territorios y sus desarrollos. Comprende la geodiversidad territorial y social y actúa en las dinámicas espaciales, justificando intervenciones adaptadas a las características del lugar”. (Jurado, 2016.p.12)

Las experiencias prácticas para los estudiantes de Trabajo Social son determinantes dentro de su formación académica, aquí es donde ellos empezaran a entender la realidad fuera de los libros, generalmente solo tienen un enfoque teórico, y al momento de ejercer se les complica porque no tienen ninguna practica o idea de cómo poder intervenir en el contexto real, al respecto Jurado (2016) plantea:

"Desarrollo local es una praxis vivencial, sin la cual todo ejercicio académico quedará incompleto. El desconocimiento del entorno hace que la lógica de las intervenciones se convierta en un caso provocado que puede poner en peligro la propia existencia del sistema” (p.90).

Las solicitudes que el profesional realiza a las diferentes instituciones universitarias sean públicas o privadas, las hace basado en las políticas del país en la cual consiste en la gestión para la implementación de diferentes espacios (recreativos o centros con los que no cuentan) con el fin de satisfacer las necesidades que ellos tienen en compañía de los diferentes autoridades y representantes barriales. "Encarar un tipo de políticas capaces de estimular la creación de entornos territoriales innovadores para la concertación estratégica de actores sociales y el fomento de la creatividad productiva y empresarial local”. (Paolo, 2014. P.74)

El Trabajador Social es un agente que siempre debe estar vinculado con la sociedad de una forma más directa con el trabajo de campo y no permanecer encerrado en una oficina, ya que al están en contacto siempre con la población estará informado día a día de los diferentes cambios que le pasará a su grupo, podrá guiarlos y trabajar de una forma rápida y correcta. "Los trabajadores sociales que 
se insertan cotidianamente en poseen distintas herramientas de intervención para transformar la realidad. Pero es el sentido que las orienta lo que merece transformarse”. (Arancibia, 2014, p.65)

Procesos de intervención en comunidad. El Trabajador Social cuando realiza una investigación y diagnóstica la problemática que está afectando a la comunidad, tiene a su disposición varios modelos de intervención (ecológico, sistémico, resolución de problemas, intervención en crisis) los cuales se seleccionara el que mejor se ajuste al proceso, utilizando las diferentes herramientas que corroborara este proceso (visitas domiciliarias, encuestas, ficha social, observación, entrevistas) y son de vital ayuda para el profesional.

Es importante siempre contar con actores dinámicos y participativos, para que sean parte del proceso de intervención de una manera positiva, ya que también siempre uno se encuentra con actores que negativos que buscan boicotear lo que se está realizando, y así juntos puedan construir un plan operacional para el cumplimiento de los objetivos propuestos y llevarlos a cabo en el menor tiempo posible, "Las capacidades participativas de los actores y de las estructuras mediadoras, permita alcanzar unos objetivos comunes y predeterminados para mejorar las condiciones económicas, sociales y culturales de las comunidades, y cuyos resultados puedan ser evaluados de forma continuada". (Gutiérrez, 2014, p.23)

Investigación acción y participativa. La finalidad de la (IAP) Investigación Acción Participativa, es que el profesional de trabajo social, que va a intervenir en la comunidad cuente con las herramientas necesarias que le den una acción transformadora al proyecto que va a ejecutar, para ello debe de conocer y saber aplicar las (IAP) de forma correcta, que le permita involucrarse y ser parte de la comunidad y así poder contar con las personas que residen en ese lugar. La investigación es la primera etapa que enfrenta un trabajador social para ejecutar un proyecto, "el planteamiento general de la investigación debe responder fundamentalmente a las siguientes cuestiones: ¿Para quién y para qué se hace? ¿Quién lo hace? ¿Por qué? ¿Cómo? ¿Cuándo?” (Marti, 2000), y de ahí poder partir, para empezar a recolectar la información, pero siempre teniendo en cuenta que esa información la debemos obtener de los habitantes de la comunidad.

Aquí se debe realizar entrevistas, encuestas y cualquier otra técnica que le permita al profesional recolectar la mayor información necesaria que sirva para nutrirse de las ventajas y desventajas que posea esta comunidad, así como en este caso, lo aplicaron los estudiantes, mismo que está reflejado 
en las fichas de sistematización desde la 1.2 hasta la 1.4 y evidenciado con la ficha socioeconómica aplicada que es el anexo 2 y a su vez con las fotografías que donde se muestra la toma de datos en la comunidad. "La primera fase del trabajo de campo consiste en la realización de entrevistas semiestructuradas a representantes políticos, personal técnico y representantes asociativos". (Martí, 2000, p.83)

Diagnóstico participativo. El diagnóstico participativo, es un método en el cuales los propios miembros de la comunidad analizan las diferentes actividades, que pueden ser necesarias para poder buscar una solución a las problemáticas que les están afectando, y no dando toda la responsabilidad a los agentes externos, en este caso el trabajador social, que están realizando la intervención, este diagnóstico puede ser apoyo por los profesionales y se lo puede realizar en varias etapas para que toda la comunidad se valla integrando a dicha labor.

Dentro de la intervención comunitaria lo principal, la actividad principal es involucrar a los actores sociales (habitantes) para que sean parte del proyecto y veedores durante su ejecución, haciendo énfasis en que los resultados de la correcta aplicación serán beneficiosos para los pobladores de dicha comunidad y por esos se requiere de la participación y voluntad de cambio de todos los involucrados. "El arte comunitario se distingue por su naturaleza colaborativa. En los proyectos, el artista interviene involucrando a la comunidad en el proceso de creación y de ejecución de las obras". (Moreno, 2016, p.19)

\section{Metodología}

La metodología fue de tipo cualitativa, a partir del método de sistematización, que según Sandoval (2001), es una propuesta metodológica cuya intención es obtener un conocimiento a partir de la recuperación de la experiencia práctica que comprende justificación, objetivos, contextualización y reconstrucción de la práctica, descripción de la práctica, elaboración del discurso descriptivo de la práctica reconstruida, interpretación crítica de la práctica reconstruida y conclusiones. Como técnica para la recolección de la información fueron la observación, las entrevistas grupales y la revisión documental y como instrumentos el registro de experiencias y el diario de campo. La población la constituyeron los habitantes de la comuna "Ligüiqui". 


\section{Análisis y discusión de los resultados}

Para la interpretación de la información y datos aportados por los instrumentos utilizados, se tomaron varios aspectos de la reconstrucción de la experiencia.

En relación a la participación de la población local, se develó que cuando se inició la práctica la mayoría de las personas creían que los estudiantes iban a brindar un asistencialismo momentáneo tal cual siempre pasa y que terminando el semestre se irían y volverían a dejar a la intemperie, la población se mostró hostil y casi no quiso colaborar, pero a medida que empezó a transcurrir el tiempo, la participación fue mejorando dado a que presidenta también se interesó más en el proyecto y a medida que los ciudadanos iban conociendo la importancia y el para qué estábamos ahí, fueron colaborando de a poco, en especial los familiares de las personas que fueron identificadas con diagnóstico médico de diabetes, ya que el beneficio era para ellos.

La sostenibilidad de las actividades, en su inicio se consideraron fáciles y con bastante aplicabilidad, pero a medida en que avanzó el proyecto se volvieron más complicadas debido a que la comunidad se mostró poco interesada, y se tuvo que buscar formas de animarlos e incentivarlos para que se unan a las actividades que se iban a realizar, se las planificó y trabajó en el salón de clase, para luego ser aplicadas a la comunidad.

Ante la poca receptividad por parte de las autoridades municipales, en aportar recursos para la creación del club de diabéticos, se logró la generación de ingresos a través de una actividad recreativa social, los mismos se utilizaron para la compra del equipo médico que sirvió para detectar los niveles de glucosa e insulina en la sangre.

Se pudo constatar que la comunidad de Ligüiqui, tiene una organización que responde al modelo tradicional de conformación de los gobiernos locales, encabezadas por su directiva, la cual estaba presidida por la señora Blanca Parrales, que era la presidenta de la comunidad, junto con el resto de los colaboradores quienes eran los representantes de todos los pobladores de dicho sector, dentro de su organización local, los puestos no representan una forma de obtener una remuneración económica, sino que lo realizan de forma voluntaria con la finalidad de sacar adelante el lugar donde vive. 
La gestión del proyecto, hizo necesario tramitar diversos recursos tanto humanos como económicos para la continuidad del mismo, en los recursos humanos se consiguió el apoyo del personal médico del subcentro de San Lorenzo, para el dictado de un taller acerca de la enfermedad de diabetes, información que luego transmitimos de casa en casa a todos los habitantes de la comunidad de Ligüiqui y realizamos varios talleres con ellos y con sus familias.

\section{Conclusiones}

En el proceso académico del estudiante de Trabajo Social, tienen que vivir varias etapas para forma su carácter profesional, la intervención comunitaria les enseña una perspectiva más cercana a la realidad social que padecen los habitantes de las comunidades.

Se lograron actividades prácticas como producto de la intervención con las comunidades y los procesos que se llevaron a cabo durante la realización y ejecución del proyecto, generando nuevos conocimientos y competencias que desarrollaron los estudiantes durante el proceso de ejecución del proyecto.

Al comienzo del proyecto, los participantes no estaban tan entusiasmados con la idea de lo que se iba a realizar, pero gracias a la cooperación de los lideres barriales se pudo dar la apertura del proyecto, y cada día los mismos habitantes se fueron sumando para la ejecución del programa.

Los estudiantes de Trabajo Social de la ULEAM dentro de sus prácticas, no solo las realizan para cumplir con lo que dice el manual de prácticas de la carrera, ni con la finalidad de brindar un asistencialismo, sino para comprometerse y orientar a la comunidad durante este proceso y que juntos vayan construyendo un proyecto que beneficie a ambos, a los pobladores para cambiar un poco su estilo de vida y a los estudiantes porque van ganando experiencia de cómo será su trabajo cuando tengan que ejercerlo.

\section{Referencias Bibliográficas}

Arancibia, J. L. (2014). Recuperando la economía: entre la cuestión social y la intervención social. Cuadernos de Trabajo Social.

Costello, B. (2011). reglamento de prácticas preprofesionales y pasantías. Manta: Pennsylvania: International Institute for Restorative Practices. 
Gómez, G. F. (2016). Redes comunitarias y avances de supervisión en trabajo social. UNED Universidad Nacional de Educación a Distancia.

Gutiérrez, J. C. (2014). "Desarrollo comunitario". Revista en Cultura de la Legalidad, 206-212.

Intriago, C. A. (2015). Formulación de políticas y participación social en infancia en los gobiernos seccionales. Ecuador: ProQuest Ebook Central.

Jurado, M. J. (2016). Desarrollo local en territorios de fronteras. Huelva: Universidad de Huelva.

Marti, J. (2000). LA INVESTIGACION - ACCION PARTICIPATIVA. ESTRUCTURA Y

FASES. En J. Marti, LA INVESTIGACION - ACCION PARTICIPATIVA. ESTRUCTURA Y

FASES (págs. 73-117). España: El viejo topo.

Moreno, G. A. (2016). La mediación artística: arte para la transformación social, la inclusión social y el trabajo comunitario. Barcelona: Ediciones Octaedro, S.L.

Paolo, L. J. (2014). Hacia un desarrollo integrador.

Roselló, E. (2010). Manual para el Trabajo Social comunitario. En R. Elena, Manual para el Trabajo Social comunitario (pág. 16). Madrid: NARCEA, S.A. DE EDICIONES. 